Pobrane z czasopisma Annales I - Philosophy and Sociology http://philosophia.annales.umcs.pl Data: 26/04/2023 15:12:05

DOI: $10.17951 /$ i.2017.42.2.147

\begin{tabular}{lcr}
\hline \multicolumn{2}{c}{ A N N A LES } \\
UNIVERSITATIS M A R I A E C URIE-SK L ODOWSK A \\
LOL. XLII, 2 & SECTIO I & 2017 \\
\hline
\end{tabular}

ARTUR KOTERSKI

Universidad Maria Curie-Skłodowska, Lublin

\title{
Laudan vs. Lakatos: algunos comentarios sobre la valoración final del demarcacionismo*
}

Laudan vs. Lakatos: kilka uwag na temat ostatecznej oceny demarkacjonizmu

En 1983 Laudan publicó un artículo bajo un título un tanto malicioso, $\mathrm{La}$ defunción del problema de demarcación, sobre el ascenso y el declive -como Popper subrayó- de uno de los problemas centrales de la epistemología․ Laudan realizó un estudio de todas las definiciones de conocimiento que contaban para él como pruebas de demarcación entre ciencia y no-ciencia. Para evaluar diferentes propuestas con distintas finalidades, y enunciados en diferentes eras, necesitaba un metacriterio, y como en principio siempre es posible pedir un meta-metacriterio, un meta-meta-metacriterio y así sucesivamente ${ }^{2}$, uno que no estuviera expuesto

* Traducido por Isabel López Almodóvar.

1 L. Laudan, The Demise of Demarcation Problem, [en:] R. S. Cohen, L.L. Laudan (eds.), Physics, Philosophy and Psychoanalysis. Essays in Honor of Adolf Grünbaum, Dordrecht 1992, DOI: https://doi.org/10.1007/978-94-009-7055-7_6. Cf. K. R. Popper, Die beiden Grundprobleme der Erkenntnistheorie, Tübingen 1979, p. 4; idem, The Logic of Scientific Discovery, New York 1959, p. 34. Cf. tambien su: idem, The Postscript to the Logic of Scientific Discovery, Vol. 1: Realism and the Aim of Science, London 1996, p. 159 y 161.

2 "Mi corazón se encoge cuando la discusión deriva a los metacriterios; me dan ganas de protestar, »Primero dame el meta-metacriterio con el cual yo puedo valorar tu metacriterio «" (J.W.N. Watkins, The Propositional Content of the Popper-Lakatos Rift, [en:] G. Kampis, L. Kvasz, M. Stölzner (eds.), Appraising Lakatos. Mathematics, Methodology, and the Man, Dordrecht 2002, DOI: https://doi.org/10.1007/978-94-017-0769-5_1, pp. 3-4). Por otra parte, Lakatos, que formuló su propio metacriterio (cf. I. Lakatos, History of Science and its Rational Reconstructions, [en:] R.C. Buck, R.S. Cohen (eds.), PSA 1970. In Memory of Rudolf Carnap. Proceedings of the 1970 Biennial Meeting Philosophy of Science Association, Dordrecht 1971 [reeditado en C. Howson 
a la falacia de regresión infinita. Para evitar esto, Laudan formula un metacriterio que apela a las percepciones básicas sobre la naturaleza y el papel de la ciencia, compartido al menos por el sector culto de la civilización occidental: no hay que pensárselo mucho para afirmar que la teoría de Newton es una parcela de la ciencia, mientras que la de Däniken, no lo es. Como consecuencia, Laudan señala tres requisitos para reflejar nuestra imagen razonable de la ciencia. Juntos establecieron el que para algunos sería el metacriterio de demarcación definitivo.

El primero de los tres es el requisito de adecuación o la condición material. Necesitamos que el criterio de demarcación sea adecuado, es decir, queremos que clasifique como científicas la mayoría de las teorías consideradas como ciencia -tanto en la actualidad como en el pasado-, esto es, queremos que explique la división de cada día. Un criterio que rechace la teoría de la relatividad no sería, evidentemente, adecuado ${ }^{3}$. Sin embargo, puesto que incluso los más eminentes científicos también cometes errores no podemos aceptar todo lo que reivindicaron a lo largo de la historia como auténticas teorías científicas. Cuando el requisito de adecuación está formulado de este modo, esto nos da cierta flexibilidad con teorías como la del flogisto o la calórica, así como otras más controvertidas.

El segundo es el requisito de precisión o la condición formal. Esperamos simplemente que cualquier criterio de demarcación aceptable presente tanto la condición necesaria como la suficiente para que una teoría obtenga el estatus de ciencia. El criterio de demarcación es una definición de ciencia, por lo que este requisito debería ser bastante obvio.

Los dos requisitos tienen un carácter metodológico; una definición de ciencia debe ser lo suficientemente adecuada y precisa. En cierto sentido ambos parecen ser triviales, pero diferentes propuestas de demarcación debatidas seriamente incumplieron al menos uno de ellos, lo cual fue eruditamente demostrado en el artículo de Laudan. La naturaleza metodológica del primero de los dos requisitos -los llamaremos condiciones metodológicas- hace que sea fácil operar con ellos.

El tercero de ellos, o la condición pragmática, es más vago y menos cómodo. Este es el requisito que cualquier buen criterio de demarcación debería excluir de la ciencia: teorías de lo contrario no deseadas. Si alguien da un criterio de demarcación que satisfaga ambas condiciones metodológicas pero clasifique por ejemplo

(ed.), Method and Appraisal in the Physical Sciences. The Critical Background to Modern Science, 1800-1905, Cambridge 1976], pp. 116-117; idem, Popper on Induction and Demarcation, [en:] P.A. Schilpp (ed.), The Philosophy of Karl Popper, La Salle IL 1974, p. 250; idem, The Intellectuals' Betrayal of Reason, [en:] I. Lakatos, P.K. Feyerabend, For and Against Method. Including Lakatos's Lectures on Scientific Method and the Lakatos-Feyerabend Correspondence, Chicago 1999, pp. 397-398) estaba convencido de que no había la menor necesidad de un criterio en un nivel más alto.

3 Cf. idem, Lakatos, History of Science..., p. 111 y 128, nota 80. 
la ariosofía como ciencia, sería rechazado por la tercera condición: entendemos la ciencia, entre otras cosas, como algo provechoso para toda la especie humana. Esta aproximación parece estar en perfecta concordancia con la posición de los demarcacionistas ${ }^{4}$ destacados.

El tercer requisito tiene una naturaleza diferente de las condiciones metodológicas. Es muy difícil explicar cómo aplicarlo en casos que no sean obvios. Y Laudan ni siquiera dio tales ejemplos. No tenía que hacerlo porque los criterios que él criticó no satisfacían las condiciones metodológicas; por tanto, no tenía que preocuparse sobre cómo aplicar la pragmática. Hay, sin embargo, una teoría en el caso de que la condición pragmática intervenga: se trata de la metodología lakatosiana de los programas de investigación científica que, por extraño que parezca, no fue tratada por Laudan ${ }^{5}$.

La definición de ciencia de Lakatos está basada, en principio, en los cuatro conceptos interconectados de aceptabilidad. Estos conceptos no se aplican a las proposiciones simples, o a las hipótesis y teorías simples. Describen series de teorías llamadas programas científicos de investigación. Aunque hay cuatro tipos de aceptabilidad, para simplificar pero sin menoscabo de la metodología de Lakatos, nos limitaremos aquí a solo dos de ellos.

Aceptabilidad $_{1}$ Una serie de teorías es aceptable ${ }_{1}-\mathrm{o}$ teóricamente progresiva- si cada nueva teoría de esta serie, $T_{\mathrm{n}+1}$, explica todo lo que fue explicado por la anterior, $T_{\mathrm{n}}$, y esto tiene algún excedente de contenido empírico, a saber, esto adicionalmente genera nuevas predicciones inexistentes en $T_{\mathrm{n}}$. Cuando una serie, es decir, un programa de investigación científica, no es teóricamente progresiva, es teóricamente regresiva ${ }^{6}$.

Aceptabilidad $_{2}$. Una serie es aceptable ${ }_{2}-$ o empíricamente progresiva- si es aceptable y cada $T_{\mathrm{n}+1}$ es corroborada en test de algunos de sus excedentes de contenido empírico. Cuando una serie no es empíricamente progresiva, es calificada como empíricamente regresiva.

4 Cf. p. ej. K. R. Popper, Die beiden Grundprobleme..., p. 4; I. Lakatos, Introduction: Science and Pseudoscience, [en:] Mathematics, Science and Epistemology. Philosophical Papers, Cambridge 1978, p. I.

5 Para el desarrollo de la metodología de I. Lakatos ver su: Criticism and the Methodology of Scientific Research Programmes, "Proceedings of the Aristotelian Society" 1968, Vol. 69; Falsification and Methodology of Scientific Research Programmes, [en:] I. Lakatos, A.E. Musgrave (eds.), Criticism and the Growth of Knowledge, Cambridge 1970; History of Science...; Popper on Induction... Para una valoración crítica del criterio de demarcación ver A. Koterski, Falsyfikacjonistyczne kryteria demarkacji w XX-wiecznej filozofii nauki, Lublin 2004, cap. 4.

6 Cf. L. Laudan, Two Dogmas of Methodology, "Philosophy of Science" 1976, Vol. 43(4), DOI: https://doi.org/10.1086/288718, pp. 585-593. 
Es bien sabido que el que fuera mentor de Lakatos durante varios años exigía que los científicos declararan por adelantado qué descubrimientos empíricos podrían desestabilizar sus teorías; lo mismo exigía el propio Lakatos: aceptabilidad es una condición que una serie debe satisfacer a priori. Sin embargo, mientras Popper concedería a una teoría algún tiempo para demostrar su consistencia, para Lakatos la aceptabilidad ${ }_{2}$ es también una condición a priori. Esto es exigible con una teoría de datos innovadores especialmente diseñada por Zahar7. Un programa es aceptable (los viejos demarcacionistas dirían "científico") si es aceptable y aceptable ${ }_{2}$. Este es el criterio de demarcación de Lakatos ${ }^{8}$. Sin embargo, debido al cambio de problemática en metodología de la ciencia, el criterio de demarcación deja de trazar la diferencia entre ciencia y metafísica, como ocurría en el original, esto es, la versión popperiana de 1934, pero esto permite en cambio la comparación y la elección entre programas rivales?

7 Cf. E. G. Zahar, Why did Einstein's Programme Supersede Lorentz's? Part I, "The British Journal for the Philosophy of Science" 1973, Vol. 24(2), DOI: https://doi.org/10.1093/bjps/24.2.95, p. 103; idem, Why did Einstein's Programme Supersede Lorentz's? Part II, "The British Journal for the Philosophy of Science" 1973, Vol. 24(3), DOI: https://doi.org/10.1093/bjps/24.3.223; I. Lakatos, E. G. Zahar, Why Did Copernicus's Research Programme Supersede Ptolemy's?, [en:] R. S. Westman (ed.), The Copernican Achievement, Berkeley 1976; E. G. Zahar, Einstein's Revolution. A Study in Heuristics, La Salle IL 1989, p. 16; M. Motterlini, The Value of Novelty, [en:] I. Lakatos, P.K. Feyerabend, op. cit. Ver también M. Schlick, Causality in the Contemporary Physics, [en:] Philosophical Papers, Vol. 2: (1925-1936), Dordrecht 1979, p. 185.

8 Esto es otra simplificación. De hecho, Lakatos no demanda un requisito tan intuitivamente inaceptable como para que el incremento en el contenido de cada nueva teoría de una serie deba ser corroborada. Por tanto, de acuerdo con Lakatos, cualquier serie aceptable debería ser consistente-

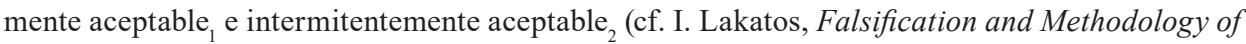
Scientific Research..., p. 134).

9 En la teoría de Lakatos la metafísica se encuentra en el mismo corazón de la ciencia; la "metafísica" tiene allí un significado múltiple (cf. A. Koterski, The Rise and Fall of Falsificationism in the Light of Neurath's Criticism, [en:] D. Dieks, W.J. Gonzalez, S. Hartmann, Th. Uebel, M. Weber [eds.], Explanation, Prediction, and Confirmation. New Trends and Old Ones Reconsidered, Dordrecht 2011, p. 497). Naturalmente, el escepticismo de Laudan sobre demarcación debe ser superado solo si tal cambio de problemática es aceptado; por el contrario, Lakatos sería solo el sepulturero del demarcacionismo. Sin embargo, como en la metodología de Lakatos la metafísica no tiene que ser pseudocientífica, las posibles preocupaciones de una antigua escuela demarcacionista son realmente innecesarias. Neurath ya subrayó el carácter empírico del pensamiento mágico que, a su vez, hacía que en principio no se pudiera distinguir de la ciencia, al menos no del modo conocido en Logik der Forschung de Popper (cf. O. Neurath, Ways of Scientific World-Conception, [en:] Philosophical Papers 1913-1946, Dordrecht 1983; idem, Magie und Technik, [en:] Gesammelte philosophische und methodologische Schriften, Bd. 1-2, Wien 1981; ver también idem, Pseudorationalism of Falsification, [en:] Philosophical Papers 1913-1946). Neurath también señaló la importancia del pensamiento precientífico para el desarrollo de la ciencia (cf. idem, Unified Science as Encyclopedic Integration, [en:] O. Neurath, R. Carnap, Ch.W. Morris (eds.), Foundations of the Unity of Science. Toward an International Encyclopedia of Unified Science, Vol. 1, Nos. 1-10, Chicago 1971, p. 21; idem, 
Laudan vs. Lakatos: algunos comentarios sobre la valoración final del demarcacionismo 151

Hay estudios de caso que sustentan la afirmación de que el criterio de demarcación de Lakatos cumple la condición de adecuación ${ }^{10}$. Bueno, es cierto que a veces las reconstrucciones lakatosianas requieren algunas acrobacias arriesgadas, pero él dice que la historia real solo será siempre una caricatura de la reconstruida ${ }^{11}$. Si aceptamos que su política es al menos tolerable, entonces su definición de ciencia satisface la condición material ${ }^{12}$.

El criterio de demarcación de Lakatos también especifica tanto la condición necesaria como la suficiente, concretamente aceptabilidad ${ }_{1}$ aceptabilidad $_{2}$, para que una teoría adquiera estatus científico. Por tanto, los requisitos metodológicos están cumplidos. ¿Pero qué ocurre con el pragmático?

En los años setenta, Lakatos estaba profundamente interesado en el debate sobre la inteligencia y la medición del CI (coeficiente intelectual). Encomendó a uno de sus estudiantes escribir un artículo para mostrar que uno de los programas competitivos del debate en cuestión era progresivo desde el punto de vista de la metodología de los programas de investigación científica. Por consiguiente, él aplicó claramente su propio criterio de demarcación a las series competitivas y finalmente encontró el ganador.

The New Encyclopedia, [en:] B. McGuinness (ed.), Unified Science. The Vienna Circle monograph series originally edited by Otto Neurath, now in English edition, Dordrecht 1987, p. 138). Por otra parte, él advirtió de que la pseudociencia o la metafísica podía ser y en realidad fue creada en lenguajes empíricos (cf. idem, Der Logische Empirismus und der Wiener Kreis, [en:] Gesammelte philosophische..., p. 746; idem, Unified Science as..., p. 19; idem, Die Gefahr sorgloser Terminologie, [en:] Gesammelte philosophische..., p. 924). Por tanto, criterios no fundamentados lógicamente pueden demarcar la ciencia de la "metafísica" de un modo suficientemente adecuado (tal como requiere la primera condición de Laudan).

10 P. ej. C. Howson (ed.), op. cit.; J. Latsis Spiro (ed.), Method and Appraisal in Economics, Cambridge 1976, DOI: https://doi.org/10.1017/CBO9780511572203.

11 Cf. I. Lakatos, Changes in the Problem of Inductive Logic, [en:] Mathematics, Science and Epistemology..., p. 178, nota 2-3; idem, Falsification and Methodology of Scientific Research, p. 146; idem, History of Science..., p. 122.

12 Ver, no obstante, los comentarios sobre tal historiografía de prominentes filósofos e historiadores de la ciencia, incluido Larry Laudan: T.S. Kuhn, Reflections on my Critics, [en:] I. Lakatos, A.E. Musgrave (eds.), op. cit., p. 256, nota 1; idem, Notes on Lakatos, [en:] R.C. Buck, R. S. Cohen (eds.), op. cit., p. 143; G. Holton, Being Caught between Dionysians and Appollonians, "Dedalus" 1974, Vol. 103, p. 68; idem, The Scientific Imagination: Case Studies, Cambridge 1978, p. 106; N. Koertge, Rational Reconstructions, [en:] R. S. Cohen, P.K. Feyerabend, M.W. Wartofsky (eds.), Essays in Memory of Imre Lakatos, Dordrecht 1976, DOI: https://doi.org/10.1007/978-94-010-14519_22, p. 362; L. Laudan, Progress and Its Problems. Towards a New Theory of Scientific Growth, Berkeley 1977, p. 169; I. Hacking, Imre Lakatos's Philosophy of Science, "The British Journal for the Philosophy of Science" 1979, Vol. 30(4), DOI: https://doi.org/10.1093/bjps/30.4.381, pp. 382-383; D.C. Stove, Cole Porter and Karl Popper: The Jazz Age in the Philosophy of Science, [en:] Against the Idols of the Age, New Brunswick 1999, pp. 11-12. 
El artículo lo escribió Peter Urbach ${ }^{13}$. En la reconstrucción del debate de Urbach hay dos programas de investigación competitivos en el origen y en el desarrollo de la inteligencia humana: el hereditario y el ambiental ${ }^{14}$. Ambos aseguran que los seres humanos están dotados con una inteligencia general innata y sus defensores aceptan el dato de que hay diferencias en cuanto a la media del CI entre diferentes grupos sociales, incluido los "grupos raciales". De acuerdo con el programa hereditarista, estas diferencias existen por razones genéticas. Los ambientalistas aseguran que la inteligencia innata alcanza la misma media para todos como grupos, y que las diferencias observadas hay que explicarlas mediante factores ambientales, como la crianza de los niños o su educación. Para los defensores de la herencia, pueden realizar cualquier papel solo si el impacto ambiental está en correlación o en armonía con los factores genéticos.

Urbach dedicó un largo y detallado artículo a demostrar que el programa ambiental era empíricamente regresivo, mientras que su rival fue el progresivo. Para conseguirlo, subrayemos de nuevo, aplicó la metodología de Lakatos y su criterio de demarcación. Por consiguiente, ya que en aquel tiempo el análisis de Urbach no fue cuestionado (al menos no públicamente) por los lakatosianos, cualquiera que aceptara la metodología de los programas de investigación científica a mediados de los setenta debía considerar el programa hereditarista como científico y exigirlo habría sido respaldado por las instituciones científicas y estatales ${ }^{15}$.

Sin embargo, uno no tarda mucho, especialmente 40 años más tarde, en preguntarse si este programa no era racista, o, en nuestro caso, si el criterio de Lakatos cumple la condición pragmática.

Aunque hay numerosas definiciones de racismo, tomaremos la de un libro muy popular, New Dictionary of Cultural Literacy. Describe el racismo como "la creencia de que algunas razas son inherentemente superiores (física, intelectual o culturalmente) a otras y, por lo tanto, tienen derecho a dominarlas" ${ }^{\prime 16}$. La obje-

13 P. Urbach, Progress and Degeneration in the "I. Q. Debate", p. I, "The British Journal for the Philosophy of Science" 1974, Vol. 25(2), DOI: https://doi.org/10.1093/bjps/25.2.99.

14 Urbach identifica el origen del programa hereditario con los trabajos de Galton en la segunda mitad del s. XIX, mientras que el "ambientalismo" empieza con J.B. Watson. Señalar tal ancestro fue probablemente para convencer al lector de que había dos tradiciones de investigación pero se deja abierto si las teorías evaluadas por Urbach están completamente desarrolladas: si no lo están, el análisis de Urbach fue prematuro. El debate sobre CI aún continúa, aportando cada vez más controversias con libros como The Bell Curve (R.J. Herrnstein, Ch. Murray, The Bell Curve. Intelligence and Class Structure in American Life, New York 1994; ver también J.P. Rushton, A.R. Jensen, 30 Years of Research on Race Differences in Cognitive Ability, "Psychology, Public Policy, and Law" 2005, Vol. 11(2), DOI: https://doi.org/10.1037/1076-8971.11.2.235).

15 Cf. I. Lakatos, History of Science..., pp. 104-105.

16 Mientras la definición presentada anteriormente es suficiente para inducir graves preocupaciones sobre posibles implicaciones del artículo de Urbach, así como el programa hereditario, el 
ción contra los programas hereditaristas y sus seguidores es que mientras que esto explica las diferencias entre la media del nivel de CI en los llamados grupos raciales en el nivel genético, también defiende la tesis de que algunos "grupos raciales" están esencialmente mejor dotados intelectualmente que otros y, por esto, ofrece claramente un argumento científico a los racistas: si la inteligencia innata es más baja en el caso de uno de los grupos investigados, ese grupo no solo es peor, sino quizá incluso menos humano. Naturalmente, la búsqueda de evidencias científicas para las teorías racistas no es nada nuevo, pero es siempre alarmante ${ }^{17}$.

Por consiguiente, este caso muestra que uno puede tener serias dudas acerca de que el criterio de demarcación de Lakatos sea aceptable. Si la conclusión anterior es acertada, entonces el criterio de Lakatos debería ser rechazado de acuerdo con el metacriterio especificado por Laudan. Si es así, su escepticismo sobre demarcación estaría justificado de nuevo ${ }^{18}$. Si, por el contrario, no estamos firmemente convencidos de la finalidad de Laudan y no queremos rechazar el mejor criterio de demarcación disponible, entonces podríamos modificar el metacriterio. ¿Existe una forma segura de hacerlo?

Al abogar por el rechazo del criterio de Lakatos, podríamos decir, en primer lugar, que deberíamos eludir una teoría de la ciencia que defiende teorías racistas; $\mathrm{y}$, en segundo lugar, que cambiar el metacriterio parece ser una maniobra ad hoc, puesto que hemos aceptado anteriormente que presentaba unas percepciones muy básicas de la ciencia.

Al abogar por las modificaciones del metacriterio de Laudan, podríamos apreciar que no todas las percepciones están representadas aquí. En su artículo, Laudan

fenómeno descrito como «racismo» es mucho más complejo y trasciende su dimensión de biólogo (véase T. Uebel, History of Philosophy of Science and the Politics of Race and Ethnic Exclusion, [en:] M. Heidelberger, M. Stadler (Hrsg.), Wissenschaftsphilosophie und Politik, Wien 2003, pp. 91-97, 106-114).

17 Algunos intentos de justificación científica del racismo son presentados por Uebel (ibídem). Cabe señalar que Urbach era consciente de cómo muchos entenderían la tesis que apoyó y en el último pasaje de su artículo dijo que estaba interesado en la verdad y no en cuestiones políticas. De alguna manera es irónico, porque, como buen lakatosiano, Urbach sabe que todas las teorías científicas son falsas.

18 Debido a que la búsqueda de demarcación falla, Laudan concluye que se trata de un pseudoproblema. Sin embargo, cada fracaso en la búsqueda de demarcación sería más bien una prueba de que no es un pseudoproblema; ya que existen algunas respuestas, aun con sus fallos, el problema debe tener respuesta. Y es, al menos en su estado original, es decir, en la versión popperiana, cuando se trata de establecer una convención adecuada. Según Laudan, sin embargo, un demarcacionista no debería haber buscado una convención, sino "rasgos epistémicos que demarcan la ciencia de otros tipos de creencias y actividades" (I. Laudan, The Demise of Demarcation..., p. 111; ver también su idem, Methodology's Prospects, [en:] A.I. Fine, P.K. Machamer (eds.), PSA 1986. Proceedings of the 1986 Biennial Meeting of the Philosophy of Science Association, Vol. 2, East Lansing 1987, pp. 348, 350, 352-353). 
Pobrane z czasopisma Annales I - Philosophy and Sociology http://philosophia.annales.umcs.pl Data: 26/04/2023 15:12:05

considera supuestas tentativas de demarcación por parte de Platón o Aristóteles, aunque no tienen nada que ver con la ciencia en el sentido moderno de la palabra, pero ni siquiera menciona a Lakatos, que fue un verdadero demarcacionista. Si Laudan había considerado la metodología de los programas de investigación científica, la necesidad de una modificación del metacriterio habría resultado evidente. Hoy en día es normal que se requiera que el criterio de demarcación permita la comparación de teorías o programas rivales. No basta con saber que las teorías de Ptolomeo y de Copérnico eran científicas; queremos saber por qué razón al elegir el sistema heliocéntrico los científicos actuaron de manera racional. La filosofía de la ciencia implica que hay y que debería haber rivalidad en la ciencia, y que los filósofos necesitan una herramienta para evaluar tales competiciones. Esta es otra percepción bien fundamentada de la ciencia y su desarrollo, y esto debería estar representado en el metacriterio.

Así, hay una manera de modificar el metacriterio de manera no ad hoc haciéndolo más amplio. Sin embargo, queremos eliminar también parte de esto. La condición pragmática es tan vaga que siempre obstruirá la ciencia en cuanto se dé la interpretación adecuada. Se trata más bien de una herramienta política, cuyo uso es mejor ilustrado por la historia de la ciencia en la Unión Soviética o en el Tercer Reich. Una vez eliminado esto, queda un criterio de demarcación bastante bueno.

Sin embargo, ¿la eliminación de la condición pragmática equivale a una aprobación abierta de cualquier tipo de investigación en ciencia, incluidos todos los tipos de investigaciones en seres humanos? $\mathrm{O}$, más generalmente, ¿es moralmente aceptable en una sociedad democrática abandonarlo?

Es una cuestión verdaderamente dramática y no hay respuestas sencillas. Incluso si mantenemos la condición pragmática, seguimos sin saber cómo usarla objetivamente $^{19}$. Las respuestas a preguntas como estas requieren decisiones, y las decisiones a menudo se toman por votación o por referencia a motivos auxiliares. Aunque la tarea de la filosofía de la ciencia es decir qué teoría es mejor en términos de parámetros específicos, la tarea del control democrático sobre la ciencia se encuentra en otra parte. Si estamos de acuerdo con esto, no necesitamos la condición pragmática en la teoría de la ciencia.

Resumamos lo dicho hasta ahora. Examinamos el criterio de demarcación de Lakatos con el metacriterio de Laudan. El resultado fue negativo. Sin embargo, en la discusión sobre la teoría de demarcación de Lakatos, advertimos un requisito que debía ser añadido al metacriterio, concretamente el requisito de comparabilidad del grado de progresividad de las teorías empíricas. Por tanto, añadimos este requisito. Al hacer esto, entendimos que el metacriterio que considerábamos el definitivo,

19 Esto se ve tal vez de una manera más clara en el caso de investigaciones de uso dual. 
de hecho era variable. En tal caso, aprovechamos la oportunidad para desechar la condición pragmática a causa de su potencialmente peligrosa vaguedad, ya que la eliminación resulta con mayor elegancia del metacriterio y, finalmente, porque el criterio pragmático rechaza el único criterio de demarcación factible, ofrecido por la metodología de los programas de investigación científica de Lakatos ${ }^{20}$.

Aunque la ampliación del metacriterio no fue problemática, el rechazo de la condición pragmática puede ser refutado de la siguiente manera: ya que lo eliminamos para salvar el criterio de demarcación de Lakatos, que, a cambio, clasifica una teoría racista como científica, también aceptamos el figurado apoyo científico del racismo. ¿De verdad tenemos que vivir con esto?

Por una parte consideramos que los resultados de las investigaciones científicas pueden tener también consecuencias indeseadas por cuestiones sociales. Cuando presentamos dos desiderata, para que la ciencia por una parte busque la verdad, por otra, en cambio, sea útil socialmente, deberíamos recordar que su constante cumplimiento no lo garantiza ninguna lógica ni ley de la naturaleza ${ }^{21}$. Es posible, como mucho, definir convencionalmente con qué fin debe ser ejercida la ciencia y a continuación, actuar según lo acordado. La aceptación de una opción determinada, aunque siempre va a tener carácter convencional, no puede ser arbitraria.

Por otra parte, sin embargo, no creemos que la conclusión extraída del artículo de Urbach con respecto al "apoyo científico" del racismo esté acreditada. Es posible que en alguna etapa del debate sobre la manera de medir el CI sus resultados compartan el apoyo empírico de cierta teoría descriptiva de la "raza", en tanto este término posea algún significado empírico. No obstante, el racismo tiene un carácter normativo, así que la declaración de apoyo científico y filosófico que resulta del debate sobre CI y su medición así como del análisis de Urbach, está basada en un falacia naturalista ${ }^{22}$. Las investigaciones presentadas por los partidarios de la teoría hereditarista, desde Galton hasta el recientemente fallecido Jensen, no otorgan ningún apoyo empírico a ninguna concepción normativa, ni a ninguna postura.

20 Es factible en términos del metacriterio de Laudan, por lo menos; sin embargo, puede haber otras razones para cuestionar la definición de Lakatos de ciencia (cf. P. Urbach, The Bayesian Alternative to the Methodology of Scientific Research Programmes, [en:] K. Gavroglu, Y. Goudaroulis, P. Nicolacopoulos (eds.), Imre Lakatos and Theories of Scientific Change, Dordrecht 1989, DOI: https://doi.org/10.1007/978-94-009-3025-4_29, p. 400).

${ }^{21}$ Algunos escépticos bioéticos afirman que debemos abandonar, entre otras cosas, la creencia de que la ciencia trata exclusivamente de cosas buenas y aceptar la verdad menos romántica de que produce asimismo lo malo. Arthur Caplan llegó a decir que en la era tecno-terrorista, al final nos mataría la ciencia.

22 También hay que tener en cuenta que como buen lakatosiano Urbach declaró no solo que ambos programas competitivos son falsos, sino que también destacó que el hasta entonces fracaso del programa ambiental no significa ni mucho menos su caída final. 
El hecho de que algunos reconozcan todo lo contrario solo muestra, una vez más, que Philipp Frank tenía razón: Según él, la enseñanza de la filosofía de la ciencia constituye una importante contribución al refuerzo de la democracia.

\section{BIBLIOGR AFÍA}

Hacking I., Imre Lakatos's Philosophy of Science, "The British Journal for the Philosophy of Science" 1979, Vol. 30(4), DOI: https://doi.org/10.1093/bjps/30.4.381.

Herrnstein R.J., Murray Ch., The Bell Curve. Intelligence and Class Structure in American Life, New York 1994.

Holton G., Being Caught between Dionysians and Appollonians, "Dedalus" 1974, Vol. 103.

Holton G., The Scientific Imagination: Case Studies, Cambridge 1978.

Howson C. (ed.), Method and Appraisal in the Physical Sciences. The Critical Background to Modern Science, 1800-1905, Cambridge 1976, DOI: https://doi.org/10.1017/CBO9780511760013.

Koertge N., Rational Reconstructions, [en:] R. S. Cohen, P.K. Feyerabend, M.W. Wartofsky (eds.), Essays in Memory of Imre Lakatos, Dordrecht 1976,

DOI: https://doi.org/10.1007/978-94-010-1451-9_22.

Koterski A., Falsyfikacjonistyczne kryteria demarkacji w XX-wiecznej filozofii nauki, Lublin 2004.

Koterski A., The Rise and Fall of Falsificationism in the Light of Neurath's Criticism, [en:] D. Dieks, W.J. Gonzalez, S. Hartmann, Th. Uebel, M. Weber (eds.), Explanation, Prediction, and Confirmation. New Trends and Old Ones Reconsidered, Dordrecht 2011.

Kuhn T.S., Notes on Lakatos, [en:] R.C. Buck, R.S. Cohen (eds.), PSA 1970. In Memory of Rudolf Carnap. Proceedings of the 1970 Biennial Meeting Philosophy of Science Association, Dordrecht 1971.

Kuhn T.S., Reflections on my Critics, [en:] I. Lakatos, A.E. Musgrave (eds.), Criticism and the Growth of Knowledge, Cambridge 1970.

Lakatos I., Changes in the Problem of Inductive Logic, [en:] Mathematics, Science and Epistemology. Philosophical Papers, Cambridge 1978.

Lakatos I., Criticism and the Methodology of Scientific Research Programmes, "Proceedings of the Aristotelian Society" 1968, Vol. 69.

Lakatos I., Falsification and Methodology of Scientific Research Programmes, [en:] I. Lakatos, A.E. Musgrave (eds.), Criticism and the Growth of Knowledge, Cambridge 1970.

Lakatos I., History of Science and its Rational Reconstructions, [en:] R.C. Buck, R.S. Cohen (eds.), PSA 1970. In Memory of Rudolf Carnap. Proceedings of the 1970 Biennial Meeting Philosophy of Science Association, Dordrecht 1971 (reeditado en C. Howson (ed.), Method and Appraisal in the Physical Sciences. The Critical Background to Modern Science, 1800-1905, Cambridge 1976).

Lakatos I., Introduction: Science and Pseudoscience, [en:] Mathematics, Science and Epistemology. Philosophical Papers, Cambridge 1978.

Lakatos I., Popper on Induction and Demarcation, [en:] P. A. Schilpp (ed.), The Philosophy of Karl Popper, La Salle IL 1974.

Lakatos I., The Intellectuals' Betrayal of Reason, [en:] I. Lakatos, P.K. Feyerabend, For and Against Method. Including Lakatos's Lectures on Scientific Method and the Lakatos-Feyerabend Correspondence, Chicago 1999.

Lakatos I., Zahar E. G., Why Did Copernicus's Research Programme Supersede Ptolemy's?, [en:] R.S. Westman (ed.), The Copernican Achievement, Berkeley 1976. 
Laudan vs. Lakatos: algunos comentarios sobre la valoración final del demarcacionismo 157

Latsis Spiro J. (ed.), Method and Appraisal in Economics, Cambridge 1976, DOI: https://doi.org/10.1017/CBO9780511572203.

Laudan L., Methodology's Prospects, [en:] A.I. Fine, P.K. Machamer (eds.), PSA 1986. Proceedings of the 1986 Biennial Meeting of the Philosophy of Science Association, Vol. 2, East Lansing 1987.

Laudan L., Progress and Its Problems. Towards a New Theory of Scientific Growth, Berkeley 1977.

Laudan L., The Demise of Demarcation Problem, [en:] R. S. Cohen, L.L. Laudan (eds.), Physics, Philosophy and Psychoanalysis. Essays in Honor of Adolf Grünbaum, Dordrecht 1992, DOI: https://doi.org/10.1007/978-94-009-7055-7_6.

Laudan L., Two Dogmas of Methodology, "Philosophy of Science" 1976, Vol. 43(4), DOI: https://doi.org/10.1086/288718.

Motterlini M., The Value of Novelty, [en:] I. Lakatos, P.K. Feyerabend, For and Against Method. Including Lakatos's Lectures on Scientific Method and the Lakatos-Feyerabend Correspondence, Chicago 1999.

Neurath O., Der Logische Empirismus und der Wiener Kreis, [en:] Gesammelte philosophische und methodologische Schriften, Bd. 1-2, Wien 1981.

Neurath O., Die Gefahr sorgloser Terminologie, [en:] Gesammelte philosophische und methodologische Schriften, Bd. 1-2, Wien 1981.

Neurath O., Magie und Technik, [en:] Gesammelte philosophische und methodologische Schriften, Bd. 1-2, Wien 1981.

Neurath O., Pseudorationalism of Falsification, [en:] Philosophical Papers 1913-1946, Dordrecht 1983.

Neurath O., The New Encyclopedia, [en:] B. McGuinness (ed.), Unified Science. The Vienna Circle monograph series originally edited by Otto Neurath, now in English edition, Dordrecht 1987.

Neurath O., Unified Science as Encyclopedic Integration, [en:] O. Neurath, R. Carnap, Ch.W. Morris (eds.), Foundations of the Unity of Science. Toward an International Encyclopedia of Unified Science, Vol. 1, Nos. 1-10, Chicago 1971.

Neurath O., Ways of Scientific World-Conception, [en:] Philosophical Papers 1913-1946, Dordrecht 1983.

Popper K.R., Die beiden Grundprobleme der Erkenntnistheorie, Tübingen 1979.

Popper K. R., The Logic of Scientific Discovery, New York 1959.

Popper K.R., The Postscript to the Logic of Scientific Discovery, Vol. 1: Realism and the Aim of Science, London 1996.

Rushton J.P., Jensen A.R., 30 Years of Research on Race Differences in Cognitive Ability, "Psychology, Public Policy, and Law" 2005, Vol. 11(2), DOI: https://doi.org/10.1037/1076-8971.11.2.235.

Schlick M., Causality in the Contemporary Physics, [en:] Philosophical Papers, Vol. 2: (1925-1936), Dordrecht 1979.

Stove D.C., Cole Porter and Karl Popper: The Jazz Age in the Philosophy of Science, [en:] Against the Idols of the Age, New Brunswick 1999.

Uebel T., History of Philosophy of Science and the Politics of Race and Ethnic Exclusion, [en:] M. Heidelberger, M. Stadler (Hrsg.), Wissenschaftsphilosophie und Politik, Wien 2003.

Urbach P., Progress and Degeneration in the "I. Q. Debate", p. I, "The British Journal for the Philosophy of Science" 1974, Vol. 25(2), DOI: https://doi.org/10.1093/bjps/25.2.99.

Urbach P., The Bayesian Alternative to the Methodology of Scientific Research Programmes, [en:] K. Gavroglu, Y. Goudaroulis, P. Nicolacopoulos (eds.), Imre Lakatos and Theories of Scientific Change, Dordrecht 1989, DOI: https://doi.org/10.1007/978-94-009-3025-4_29.

Watkins J.W.N., The Propositional Content of the Popper - Lakatos Rift, [en:] G. Kampis, L. Kvasz, M. Stölzner (eds.), Appraising Lakatos. Mathematics, Methodology, and the Man, Dordrecht 2002, DOI: https://doi.org/10.1007/978-94-017-0769-5_1. 
Zahar E. G., Einstein's Revolution. A Study in Heuristics, La Salle IL 1989.

Zahar E. G., Why did Einstein's Programme Supersede Lorentz's? Part I, "The British Journal for the Philosophy of Science" 1973, Vol. 24(2), DOI: https://doi.org/10.1093/bjps/24.2.95.

Zahar E.G., Why did Einstein's Programme Supersede Lorentz's? Part II, "The British Journal for the Philosophy of Science" 1973, Vol. 24(3), DOI: https://doi.org/10.1093/bjps/24.3.223.

\section{SUMMARY}

The paper reconstructs and examines the meta-criterion of demarcation between science and pseudo-science as proposed by Laudan (1983). The analysis shows that Laudan's meta-criterion overlooks a crucial demand that any reliable criterion should satisfy. This, in turn, opens up a possibility of a non-ad hoc modification of Laudan's proposal. When the meta-criterion finally meets the requirement which states that any reliable criterion of demarcation has to enable a comparison of any two scientific theories with regard to the degree of their progressiveness, it is possible to point out at least one advanced theory which gives an acceptable definition of science, i.e. Lakatos's methodology of scientific research programs - strangely enough, not attended to by Laudan. Therefore, the skeptical conclusions of his paper can be refuted; furthermore, after such a modification the meta-criterion of demarcation acquires a homogenous form what makes it stable in a way originally intended by Laudan.

Keywords: problem of demarcation; meta-criterion of demarcation; methodology of scientific research programs

\section{STRESZCZENIE}

Celem artykułu jest rekonstrukcja i analiza przedstawionego przez Laudana (1983) metakryterium demarkacji między nauką a pseudonauką. Okazuje się, że Laudan przeoczył kluczowy warunek, który powinno uwzględniać każde godne uwagi metakryterium. Otwiera to z kolei możliwość wprowadzenia do jego propozycji poprawek, które nie będą miały charakteru ad hoc. Kiedy tym sposobem metakryterium obejmuje również wymóg, by kryterium demarkacji umożliwiało porównanie teorii naukowych co do stopnia ich postępowości czy naukowości, można wskazać przynajmniej jedną teorię podającą akceptowalne kryterium demarkacji, mianowicie Lakatosową metodologię naukowych programów badawczych, którą w swej pracy Laudan pominął jednak milczeniem. Wtedy też można odrzucić sceptyczne wnioski jego artykułu. Dzięki tej modyfikacji metakryterium demarkacji otrzymuje dodatkowo jednolitą postać, za sprawą której staje się ono stabilne, tak jak tego pierwotnie chciał Laudan.

Słowa kluczowe: problem demarkacji; metakryterium demarkacji; metodologia naukowych programów badawczych

\section{RESUMEN}

El artículo reconstruye y examina el metacriterio de demarcación entre ciencia y pseudociencia como fue propuesto por Laudan (1983). Este análisis demuestra que el metacriterio de Laudan pasa por alto requisitos cruciales que cualquier criterio aceptable debería cumplir. Lo que a su vez posibilita una modificación no ad hoc de la propuesta de Laudan. Cuando el metacriterio finalmente se enfrenta al requisito que establece que cualquier criterio de demarcación aceptable tiene 
Pobrane z czasopisma Annales I - Philosophy and Sociology http://philosophia.annales.umcs.pl Data: 26/04/2023 15:12:05

Laudan vs. Lakatos: algunos comentarios sobre la valoración final del demarcacionismo 159

que posibilitar la comparación de dos teorías científicas cualesquiera con respecto a su grado de progresividad, es posible señalar al menos una teoría avanzada que dé una definición aceptable de ciencia, es decir, la metodología de los programas de investigación científica de Lakatos, omitidos en el artículo de Laudan, por extraño que parezca. Por esto pueden refutarse las conclusiones dudosas de su artículo; y lo que es más, tras la modificación, el metracriterio de demarcación adquiere una forma homogénea que lo hace estable, tal y como Laudan pretendía originariamente.

Palabras claves: problema de demarcación; metacriterio de demarcación; metodología de los programas de investigación científica 